\title{
Environmental factors influence the epigenetic signature of newborns from mothers with gestational diabetes
}

\author{
Emil Andersen 1(i), Ali Altıntaş ${ }^{1}$, Ulrika Andersson-Hall², Agneta Holmäng² \& Romain \\ Barrès*,1 iD \\ ${ }^{1}$ Novo Nordisk Foundation Center for Basic Metabolic Research, Faculty of Health \& Medical Sciences, University of Copenhagen, \\ Copenhagen, Denmark \\ ${ }^{2}$ Department of Physiology, Institute of Neuroscience \& Physiology, Sahlgrenska Academy at University of Gothenburg, \\ Gothenburg, Sweden \\ *Author for correspondence: Tel.: +45 353372 88; Fax. +45 353371 01; barres@sund.ku.dk
}

\begin{abstract}
Aim: To investigate the degree by which epigenetic signatures in children from mothers with gestational diabetes mellitus (GDM) are influenced by environmental factors. Methods: We profiled the DNA methylation sihgnature of blood from lean, obese and GDM mothers and their respective newborns. Results: DNA methylation profiles of mothers showed high similarity across groups, while newborns from GDM mothers showed a marked distinct epigenetic profile compared with newborns of both lean and obese mothers. Analysis of variance in DNA methylation levels between newborns showed higher variance in the GDM group. Conclusion: Our results suggest that environmental factors, rather than direct transmission of epigenetic marks from the mother, are involved in establishing the epigenetic signature associated with GDM.
\end{abstract}

First draft submitted: 22 February 2019; Accepted for publication: 18 March 2019; Published online: 10 April 2019

Keywords: DNA methylation $\bullet$ epigenetic inheritance $\bullet$ gestational diabetes $\bullet$ obesity $\bullet$ pregnancy

Pregnancy represents a critical window of development where nutritional and hormonal factors can have a major influence on disease risk later in the life of the offspring. For instance, offspring born from mothers affected by gestational diabetes mellitus (GDM) during pregnancy exhibit increased adiposity, adverse cardiometabolic risk profile, higher incidence of Type II diabetes and earlier onset of puberty among girls [1,2]. This suggests that factors in the extracellular milieu, such as hyperglycemia during fetal growth could determine metabolic health [3,4]. Recently, we showed that both maternal obesity and glycemia are determinants of increased early life adiposity, with maternal GDM being more influential than maternal weight [5]. While genetic factors may be at play, studies conducted in genetically identical animals indicate that environmental factors affect developmental programming through the regulation of epigenetic signals $[6,7]$.

Epigenetic signals include methylation of DNA, a covalent modification of DNA associated with altered transcriptional activity, which regulates gene accessibility of the transcription machinery $[8,9]$. Epigenetic patterns established during development thereby regulate gene expression and numerous fundamental embryonic processes such as genomic imprinting, $\mathrm{X}$ inactivation and the regulation of cell lineage progression and tissue specificity [10,11]. Several studies have established an association between GDM and an altered epigenetic profile in the offspring [12-19]. Others have compared the epigenetic profile of placenta to that of cord blood of the infant [20,21].

Factors such as exercise, weight loss and dietary factors can acutely remodel the epigenome of metabolic cells and tissues [22-27], providing evidence that DNA methylation is a dynamic process highly amenable to external factors. A recent report that employed simulated and empirical data from the Dutch famine suggested that selection of embryos by adverse environmental factors, and not dynamic DNA methylation changes, contribute to DNA methylation signatures in the infant [28]. Further studies are thus warranted to investigate the respective contribution 
of gametic inheritance, selection of embryos and extracellular factors during development in setting altered DNA methylation signatures observed in children born from mothers with GDM.

Here, we sought to compare the epigenetic profile of blood from mothers with GDM to that of their offspring and gain insight into the nature of the transmission of epigenetic patterns from mother to offspring. We performed reduced representation bisulfite sequencing (RRBS) in blood from eight lean, normal glucose tolerant (NGT) women, eight women with obesity and eight women with GDM as well as their respective newborns. We confirmed that DNA methylation signatures of newborns from GDM mothers are altered; however, no difference in epigenetic signatures of the blood was found between lean mothers and mothers with obesity or GDM. Additionally, we report an increased variance in DNA methylation between newborns of GDM mothers, compared with newborns of lean and obese mothers. Our results suggest that a direct transmission of epigenetic marks from the mother is not involved in establishing the epigenetic signature associated with GDM, but instead, supports that environmental influences are at play.

\section{Materials \& methods}

\section{Ethical approval}

The study was approved by the ethics committee at the University of Gothenburg (Dnr 402-08). All women received oral and written information about the study and gave informed written consent before enrollment of themselves and their children.

\section{Subjects}

As part of the pregnancy obesity nutrition child health (PONCH) study [5], lean (BMI 18.5-24.9 kg/m²) and obese $\left(B M I \geq 30 \mathrm{~kg} / \mathrm{m}^{2}\right.$ ) pregnant women were recruited from six antenatal health units within the Gothenburg area, and women with GDM (irrespective of BMI) were recruited at the Sahlgrenska University Hospital at time of diagnosis. In Gothenburg at the time of the present study (2009-2015), all pregnant women had nonfasting blood glucose measured regularly throughout pregnancy, and women with an elevated nonfasting glucose $(>8 \mathrm{mmol} / \mathrm{l})$ underwent oral glucose tolerance tests, where they were diagnosed according to the European Association for the Study of Diabetes 1991 criteria [29]. None of the women included in the present study received insulin or metformin treatment. All women (in all three groups) were born in Sweden to parents of European ethnicity. Children included in the study were divided into groups corresponding to the category assigned to their mother. Mode of delivery at birth was vaginal delivery for all children apart from one child in the obese group and one in the GDM group that were born by cesarean sections.

\section{Maternal visits}

Maternal pregnancy visits have been described previously [5]. These were all in the morning after an overnight fast, and included anthropometric measurements, blood sampling and completion of life-style questionnaires. HbA1c, C-reactive protein (CRP), low-density lipoprotein (LDL) and high-density lipoprotein (HDL) and glucose levels were analyzed at the accredited (SWEDAC ISO-15189; No.1240) Clinical Chemistry Laboratory, Sahlgrenska University Hospital at weeks 35-37 (Trimester 3 [T3]). GDM women received standard obstetric hospital care including diet and lifestyle advice. As GDM women did not attend study visits early in pregnancy, trimester one weights were collected from medical records.

\section{Infant anthropometric measurements}

Infant anthropometric measurements have been previously described [5]. Birth-weight and length were collected from medical records. Anthropometric and body composition measurements were made at visits at 1 week (410 days) and 12 weeks (80-90 days) after birth, and anthropometric measurements at 52 weeks (355-375 days). During the two first visits, weight, fat mass and fat-free mass were measured by air-displacement plethysmography using a PEAPOD (software version 3.3.0; COSMED, Rome, Italy).

\section{DNA isolation}

Blood from mothers was taken from a fasting blood sample collected in T3. Blood from infants was sampled with a neonatal heel prick $48 \mathrm{~h}$ after birth. Blood samples from both mother and infant were collected in EDTA tubes and stored at $-80^{\circ} \mathrm{C}$. 
Genomic DNA was extracted from whole blood using DNeasy Blood and Tissue Kit (QIAGEN, Hilden, Germany) according to the manufacturer's protocol. Yield and quality was assessed by Qubit dsDNA HS Assay Kit (Thermo Fisher Scientific, Hvidovre, Denmark) and NanoDrop (Thermo Fisher Scientific).

\section{Library preparation}

Multiplexed RRBS was performed as previously described [23,24]. Briefly, genomic DNA was incubated with Msp1 (NEB, Frankfurt, Germany) restriction enzyme overnight for fragmentation. Adenylation was performed using dNTP (NEB) and Klenow fragment (NEB) followed by an AMpure Bead clean-up (Beckman Coulter, Copenhagen, Denmark) and ligation to TruSeq adapters (Illumina, CA, USA). Twelve ligated samples were pooled and subjected to bisulfite conversion using EZ DNA Methylation Kit (Zymo Research, Freiburg, Germany). The library pool was amplified by PCR $\left(2 \mathrm{~min} 95^{\circ} \mathrm{C}\left[30 \mathrm{~s} 95^{\circ} \mathrm{C}, 30 \mathrm{~s} 65^{\circ} \mathrm{C}\right.\right.$; $\left.45 \mathrm{~s} 72^{\circ} \mathrm{C}\right] \times 20$ cycles; 7 min $\left.72^{\circ} \mathrm{C}\right)$ with PfuTurbo Hotstart DNA polymerase (Agilent, Glostrup, Denmark). PCR products were purified using the AMPure Bead clean-up. Libraries were sequenced on a HiSeq 2500 (Illumina) at the Danish National High-Throughput DNA Sequencing Centre, on an 81 bp single-end sequencing mode.

\section{Data analysis}

Differential methylation analysis

FASTQ files were preprocessed with Trim Galore using the -rrbs flag. Alignment and CpG coverage statistics were computed using Bismark [30]. The missing values in $\mathrm{CpG}$ sites were $\mathrm{kNN}$ imputed for both methylation and unmethylation matrix, separately [31]. Afterward, the $\mathrm{CpG}$ sites were aggregated into probe regions comprising a minimum of $20 \mathrm{CpG}$ sites, close to each other, no more than $100 \mathrm{bp}$ in at least $95 \%$ of the samples by using BiSeq [32]. SNPs and high coverage CpG sites were filtered out. A generalized linear model $(y \sim 0+$ group + gender) considering gender as a covariate was fit. The differentially methylated regions (DMRs) aggregated into gene and promoters were identified by using edgeR comprising $\mathrm{CpG}$ probes with at least 100 counts for all samples [33]. DMR analysis was performed for three different genomic regions: $\mathrm{CpG}$ probes; promoters (-1 to $2 \mathrm{~kb}$ from transcription start site [TSS]); genes. Gene set enrichment analysis was performed using FRY [34].

Mean CpG probe methylation

Mother and newborn mean CpG probe methylation levels were compared by using pairwise Wilcoxon-test, across individuals.

Correlation of $C p G$ sites

CpG probe methylation levels across samples were correlated to each other by Pearson's correlation. Correlation between clinical parameters and DMRs was performed by using Spearman correlation. The p-values for each type of correlation analysis were adjusted for multiple testing correction using Benjamini-Hochberg method [35].

\section{Cell type deconvolution}

Cell type deconvolution analysis requires a reference matrix and input matrix to be deconvoluted. BLUEPRINT [36] epigenome consortium data were retrieved using DeepBlueR (Supplementary Table 4) [37]. Mean $\beta$-values for CpG islands were used for generating the reference matrix for cell type deconvolution. The reference matrix was comprised of B cell, CD14-positive monocyte, CD4-positive T cell, CD8-positive T cell, natural killer cell and neutrophils. To identify the most informative CpG islands for the cell type deconvolution analysis, sparse PCA was performed on the reference matrix and zero loadings on PC1 was removed from the reference matrix [38]. The final reference matrix contained $4016 \mathrm{CpG}$ islands (Supplementary Table 5). The kNN imputed methylation/unmethylation matrix was used to generate the $\beta$-values and aggregated into $\mathrm{CpG}$ 's matching the genomic locations in the reference matrix. Deconvolution analysis was performed by using Houseman method [39] and EpiDISH [40].

Data availability

The Gene Expression Omnibus (GEO) accession number for the DNA-methylation datasets is GSE128289.

\section{Results}

To study the influence of obesity and GDM on the transmission of DNA methylation patterns from mother to newborn, we performed genome-wide DNA methylation analysis at single nucleotide resolution using RRBS (an 
Table 1. Clinical parameters of mothers, fathers and newborns.

\begin{tabular}{|c|c|c|c|}
\hline Parameter & Lean & Obese & GDM \\
\hline $\mathbf{N}$ & 8 & 8 & 8 \\
\hline \multicolumn{4}{|l|}{ Mothers } \\
\hline Age (years) & $27.4 \pm 3$ & $29.4 \pm 4$ & $34.1 \pm 3^{\dagger, \ddagger}$ \\
\hline GDM diagnosis (days) & $0 \pm 0$ & $0 \pm 0$ & $146 \pm 65^{\dagger}$. \\
\hline Prepregnancy BMI $\left(\mathrm{kg} / \mathrm{m}^{2}\right)$ & $21 \pm 2$ & $35.1 \pm 4^{\dagger}$ & $27.3 \pm 6^{\dagger}$ \\
\hline Tri 3 BMI $\left(\mathrm{kg} / \mathrm{m}^{2}\right)$ & $24.6 \pm 2$ & $38.9 \pm 5^{\dagger}$ & $30.5 \pm 6^{\dagger}$. \\
\hline Tri 3 gestational weight gain $(\mathrm{kg})$ & $11.8 \pm 3$ & $11 \pm 4$ & $7.3 \pm 6$ \\
\hline Tri 3 weight (kg) & $68 \pm 6$ & $106.7 \pm 14^{\dagger}$ & $81.4 \pm 12^{\dagger, \ddagger}$ \\
\hline Tri 3 fat percentage (\%) & $30.9 \pm 6$ & $45.4 \pm 6^{\dagger}$ & $34.9 \pm 6$ \\
\hline Tri $3 \mathrm{HbA} 1 \mathrm{c}(\mathrm{mmol} / \mathrm{mol})$ & $28.4 \pm 2$ & $31.6 \pm 2^{\dagger}$ & $32.8 \pm 3^{\dagger}$ \\
\hline Tri 3 HOMA-IR & $1.47 \pm 1$ & $4.09 \pm 1^{\dagger}$ & $2.23 \pm 1$ \\
\hline Tri 3 glucose $(\mathrm{mmol} / \mathrm{l})$ & $4.67 \pm 0$ & $4.59 \pm 0$ & $4.45 \pm 1$ \\
\hline Tri 3 CRP (mg/l) & $2.1 \pm 1$ & $7.5 \pm 4^{\dagger}$ & $5.1 \pm 5$ \\
\hline Tri 3 insulin (pmol/l) & $7.3 \pm 3$ & $19.9 \pm 7$ & $10.6 \pm 5$ \\
\hline Tri 3 IGF-1 ( $\mu \mathrm{g} / \mathrm{l})$ & $241 \pm 63$ & $328 \pm 98$ & $270 \pm 86$ \\
\hline Tri 3 leptin $(\mu \mathrm{g} / \mathrm{l})$ & $17.2 \pm 14$ & $53.9 \pm 18^{\dagger}$ & $21.5 \pm 7^{\ddagger}$ \\
\hline Tri 3 adiponectin (mg/l) & $17.8 \pm 13$ & $10.1 \pm 5^{\dagger}$ & $17.9 \pm 17^{\ddagger}$ \\
\hline Tri 3 TSH (mIU/l) & $1.89 \pm 1$ & $2.4 \pm 2$ & $1.59 \pm 1$ \\
\hline Tri 3 T4 (pmol/l) & $12.99 \pm 1$ & $11.69 \pm 2$ & $13.25 \pm 1$ \\
\hline Tri 3 ferritin $(\mu \mathrm{g} / \mathrm{l})$ & $15.7 \pm 8$ & $26.4 \pm 21$ & $23 \pm 7$ \\
\hline Tri 3 transferrin $(\mu \mathrm{mol} / \mathrm{l})$ & $4.32 \pm 1$ & $3.84 \pm 1$ & $4.12 \pm 1$ \\
\hline Tri 3 cholesterol (mmol/l) & $6.75 \pm 1$ & $6.14 \pm 0$ & $5.74 \pm 1$ \\
\hline Tri $3 \mathrm{HDL}(\mathrm{mmol} / \mathrm{l})$ & $2.21 \pm 1$ & $1.85 \pm 1$ & $1.75 \pm 0$ \\
\hline Tri 3 LDL (mmol/l) & $4.2 \pm 1$ & $3.69 \pm 0$ & $3.51 \pm 1$ \\
\hline \multicolumn{4}{|l|}{ Fathers } \\
\hline Age (years) & $29.9 \pm 4$ & $31.3 \pm 4$ & $34.9 \pm 3$ \\
\hline BMI $\left(\mathrm{kg} / \mathrm{m}^{2}\right)$ & $24.3 \pm 4$ & $25.3 \pm 4$ & $25.7 \pm 2$ \\
\hline \multicolumn{4}{|l|}{ Newborns } \\
\hline Males/total babies & $2 / 8$ & $7 / 8$ & $4 / 8$ \\
\hline Gestational age (weeks) & $40.5 \pm 0.7$ & $41 \pm 1$ & $39.6 \pm 1^{\ddagger}$ \\
\hline Birth height (cm) & $50.3 \pm 1.3$ & $51.6 \pm 1.8$ & $50.6 \pm 1.2$ \\
\hline Birth weight (kg) & $3.7 \pm 0.4$ & $3.8 \pm 0.4$ & $3.6 \pm 0.4$ \\
\hline Birth BMI $\left(\mathrm{kg} / \mathrm{m}^{2}\right)$ & $14.6 \pm 1$ & $14.1 \pm 1.1$ & $14.1 \pm 1.3$ \\
\hline Week 1 BMI $\left(\mathrm{kg} / \mathrm{m}^{2}\right)$ & $14.5 \pm 1.3$ & $14 \pm 1.3$ & $14.6 \pm 1.3$ \\
\hline Week 1 fat mass (\%) & $14 \pm 4.5$ & $13.5 \pm 4$ & $14.3 \pm 3.4$ \\
\hline Week 1 fat free mass (\%) & $86 \pm 4.5$ & $86.5 \pm 4$ & $85.7 \pm 3.4$ \\
\hline Week $12 \mathrm{BMI}\left(\mathrm{kg} / \mathrm{m}^{2}\right)$ & $17 \pm 1.7$ & $16.6 \pm 0.9$ & $16.7 \pm 0.4$ \\
\hline Week 12 fat mass (\%) & $24.3 \pm 5$ & $24.9 \pm 2.6$ & $29.1 \pm 2$ \\
\hline Week 12 fat free mass (\%) & $75.7 \pm 5$ & $75.1 \pm 2.6$ & $70.9 \pm 2$ \\
\hline \multicolumn{4}{|c|}{$\begin{array}{l}\text { Data are mean } \pm \text { standard deviation from the cohort. Differences between groups was analyzed by one-way ANOVA and Turkey's multiple comparisons test. } \\
t_{p}<0.05 \text { versus Control subjects in same parameter. } \\
\ddagger p<0.05 \text { versus Obese in same parameter. } \\
\text { CRP: C-reactive protein; GDM: Gestational diabetes mellitus; HDL: High-density lipoprotein; HOMA-IR: Homeostatic model assessment-insulin resistance; LDL: Low-density } \\
\text { lipoprotein; Tri: Trimester. }\end{array}$} \\
\hline
\end{tabular}

assay that targets DNA regions with a high CpG content) in blood from 24 mother-newborn pairs; eight lean, NGT women, eight women with obesity and eight women with GDM (four newborn and one mother was excluded due to low mapping efficiency [Supplementary Table 1]). Clinical characteristics of study participants are presented in Table 1. To minimize the confounding effects of paternal inheritance, fathers of all groups were matched for age and BMI (Table 1). Prepregnancy BMI were higher in both mothers from the Obese $\left(35.1 \mathrm{~kg} / \mathrm{m}^{2} \pm 4\right)$ and GDM group $\left(27.3 \mathrm{~kg} / \mathrm{m}^{2} \pm 6\right)$, compared with Lean mothers $\left(21 \mathrm{~kg} / \mathrm{m}^{2} \pm 2\right)$. Gestational weight gain was similar 
across groups, while HbA1c levels were higher in both Obese and GDM mothers. Glucose levels were controlled by lifestyle treatment in the GDM mothers, and, consequently, insulin resistance, as measured by the homeostatic model assessment (HOMA) insulin resistance (IR) method, was only higher in the Obese mothers (Table 1). Newborns across groups were clinically homogeneous, except for shorter gestational age in GDM (Table 1).

\section{Distinct epigenetic signatures in each mother-newborn pair}

Initial investigation of DNA methylation signatures in mother-newborn pairs by multidimensional scaling plotting revealed that the largest separation was due to the sex of the newborn, which has been previously reported (Supplementary Figure 1A) [13]. Including sex as a covariate corrected for the heavy sex distribution as visualized on the multidimensional scaling plot (Figure 1A). Including sex as a covariate removed otherwise significantly methylated regions on the X chromosome (Supplementary Table 2). Compared with mothers, mean CpG probe methylation levels were lower in newborns, regardless of the group, while a tendency toward a higher methylation in GDM newborns was observed, similar to a previous report (Figure 1B) [16]. At the genomic region level, DNA methylation levels in newborns compared with mothers were lower for 181 regions in the Lean group, 113 in the Obese group and 93 regions in the GDM group, while higher for only 25, 12 and 20 regions in Lean, Obese and GDM, respectively (Supplementary Table 2). A larger overlap of DMRs was found between Lean and Obese (72) compared with both Lean and GDM (50) and Obese and GDM (43) (Figure 1C). Together with a lower correlation of maternal to newborn DNA methylation (Supplementary Figure 1B), the data suggest that GDM is associated with an altered epigenetic variation between mothers and their respective babies, compared to Lean and Obese.

\section{Specific gene pathways are affected by obesity \& GDM during pregnancy}

To gain functional insight into the gene pathways differentially affected between mother and children, we performed gene ontology analysis of the genes nearest to the DMRs found between mothers and their respective newborns. We found that the majority of terms were common across groups and, noticeably, for hypomethylated regions, numerous terms related to development such as 'positive regulation of neuron projection development', 'in utero embryonic development' and 'lymph vessel development' (Figure 1D \& Supplementary Table 3). Some terms were only shared by one of two groups such as 'neuron development' and 'cell differentiation' (commonly hypomethylated in the Lean and Obese groups), 'response to nutrient levels' (only hypomethylated in Obese group) and 'positive regulation of cholesterol efflux' (only hypermethylated in the GDM group) (Figure 1D). These data reinforce that the epigenetic pattern of children can be influenced by the presence of obesity or diabetes in the mother and further suggest that altered epigenetic signature may affect particular biological processes.

\section{Epigenetic signatures are not set by different cell type compositions}

To control for the possibility that distinct epigenetic profiles werecaused by a remodeling of cell type composition under each pregnancy state, we estimated cell type composition in each samples using a newly developed method to deconvolutebased on blood cell type specific DNA methylation databases [39,40] and using either the Houseman or EpiDISH methods. Both methods returned similar cell type compositions (Supplementary Figure 1C). Analysis by the EpiDISH method revealed a clear difference between mothers and newborns, where mothers' blood showed higher counts for neutrophils, while newborns were more enriched for T lymphocytes (Figure 1E). Our results are consistent with a previous study that performed direct cell type quantification by flow cytometry of infant and adult blood samples [41]. Importantly, we did not observe any difference between groups (Figure 1E). Thus, our data suggest that differences in DNA methylation between Lean, Obese and GDM groups are independent of blood cell type composition.

\section{The altered epigenome of GDM newborns may be caused by environmental influences}

Next, we investigated whether the differences in DNA methylation that we detected between mother and newborn pairs could be due to altered epigenetic patterns of mothers or newborns. Consistent with differential methylation analysis, correlation analyses of DNA methylation levels between mothers and their respective newborn showed higher associations in the Lean group compared with the Obese and GDM groups (Supplementary Figure 2A). Comparing DNA methylation between mothers showed high association and no differential methylation across groups (Supplementary Figure 2B \& Supplementary Table 2). On the contrary, we found marked differences in DNA methylation when comparing newborns across groups, with 187 DMRs (164 hypo- and 23 hyper-methylated) 

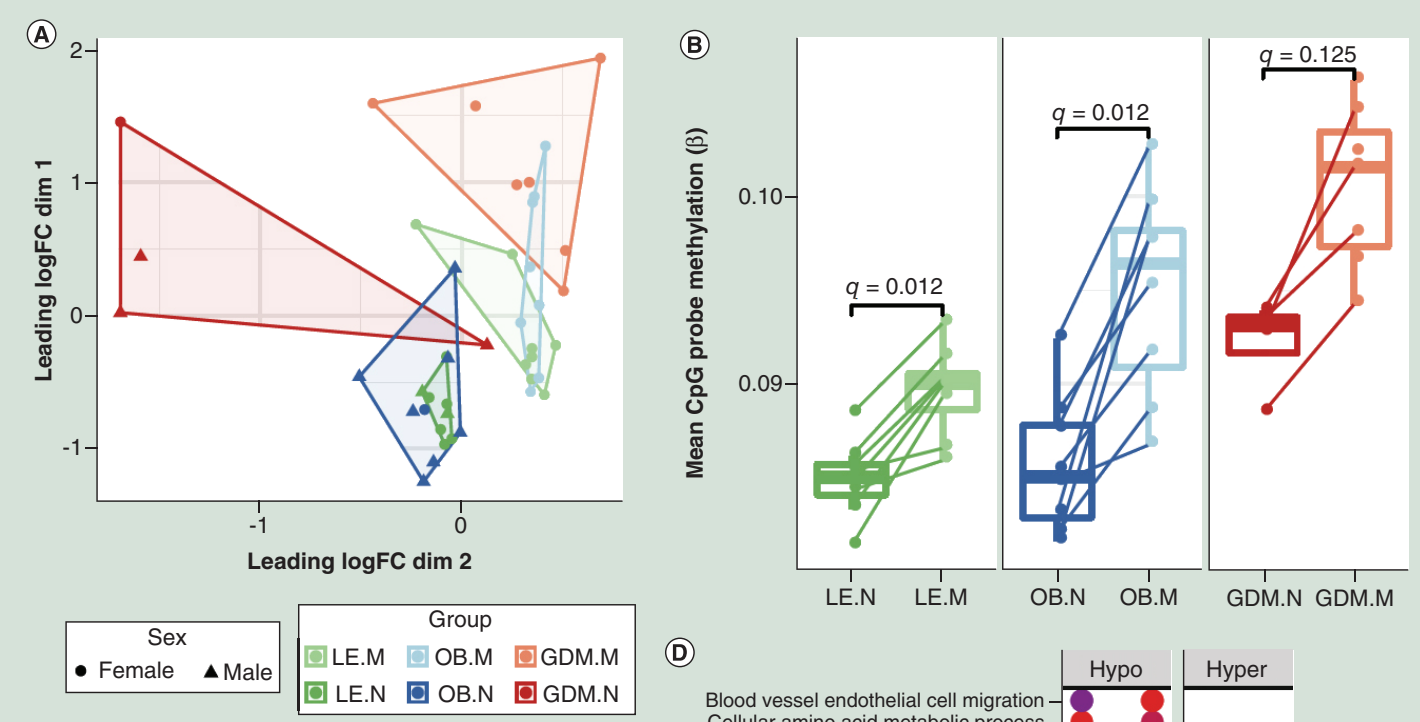

(D)

(c)
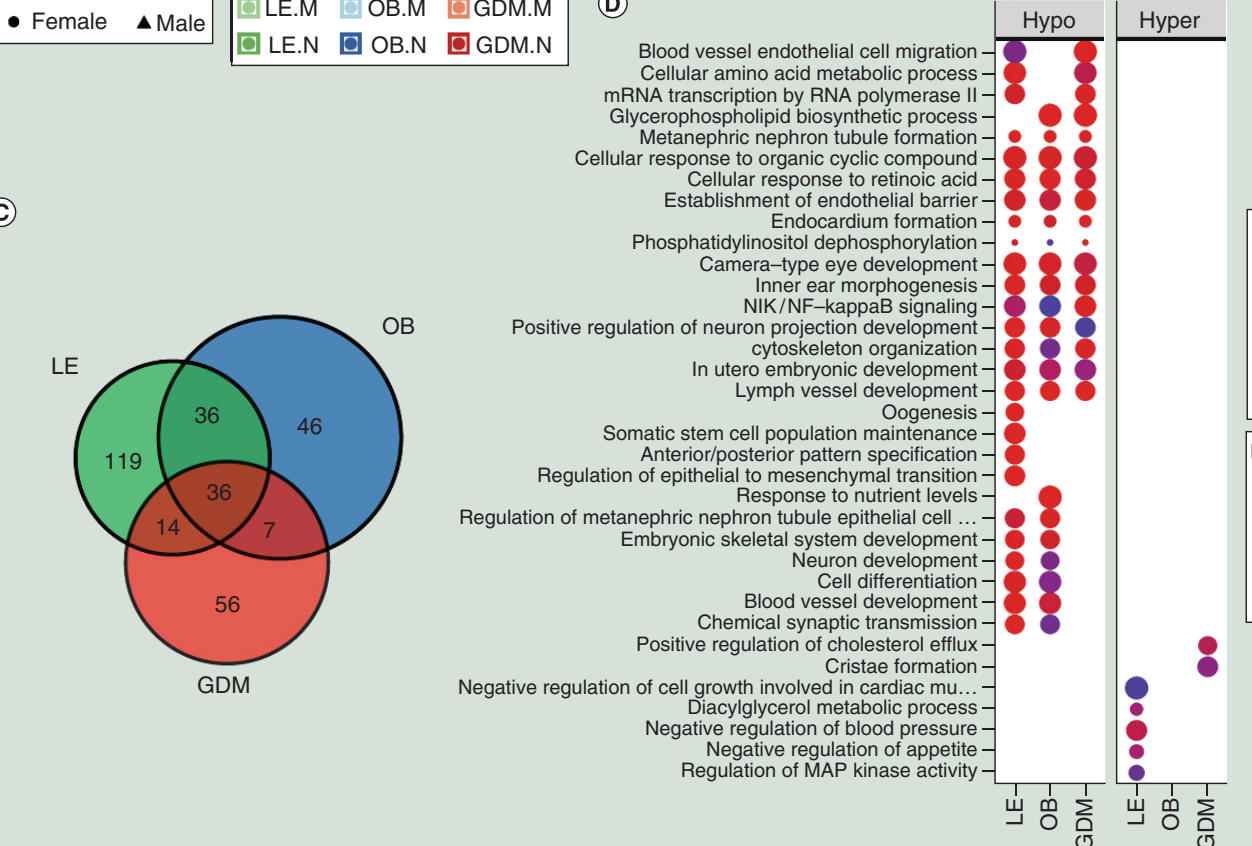

$$
\operatorname{lng}
$$

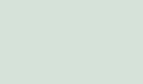$$
\begin{array}{r}
\text { Establishment of endothelial barrier } \\
\text { Endocardium formation - }
\end{array}
$$

Endocardium formation
Phosphatidylinositol dephosphorylation -

Camera-type eye development
Inner ear morphogenesis Inner ear morphogenesis
NIK/NF-kappaB signaling NIK/NF-kappaB signaling
Positive regulation of neuron projection development
cytoskeleton organization cytoskeleton organization-
In utero embryonic development Lymph vessel development Somatic stem cell population maintenance Anterior/posterior pattern specification Regulation of epithelial to mesenchymal transition Response to nutrient levels
Regulation of metanephric nephron tubule epithelial cell ... Embryonic skeletal system development Neuron development Blood vessel development Chemical synaptic transmission Positive regulation of cholesterol efflux
Cristae formation Negative regulation of cell growth involved in cardiac mu... -
Diacylglycerol metabolic process Negative regulation of blood pressure Negative regulation of appetite Regulation of MAP kinase activity -
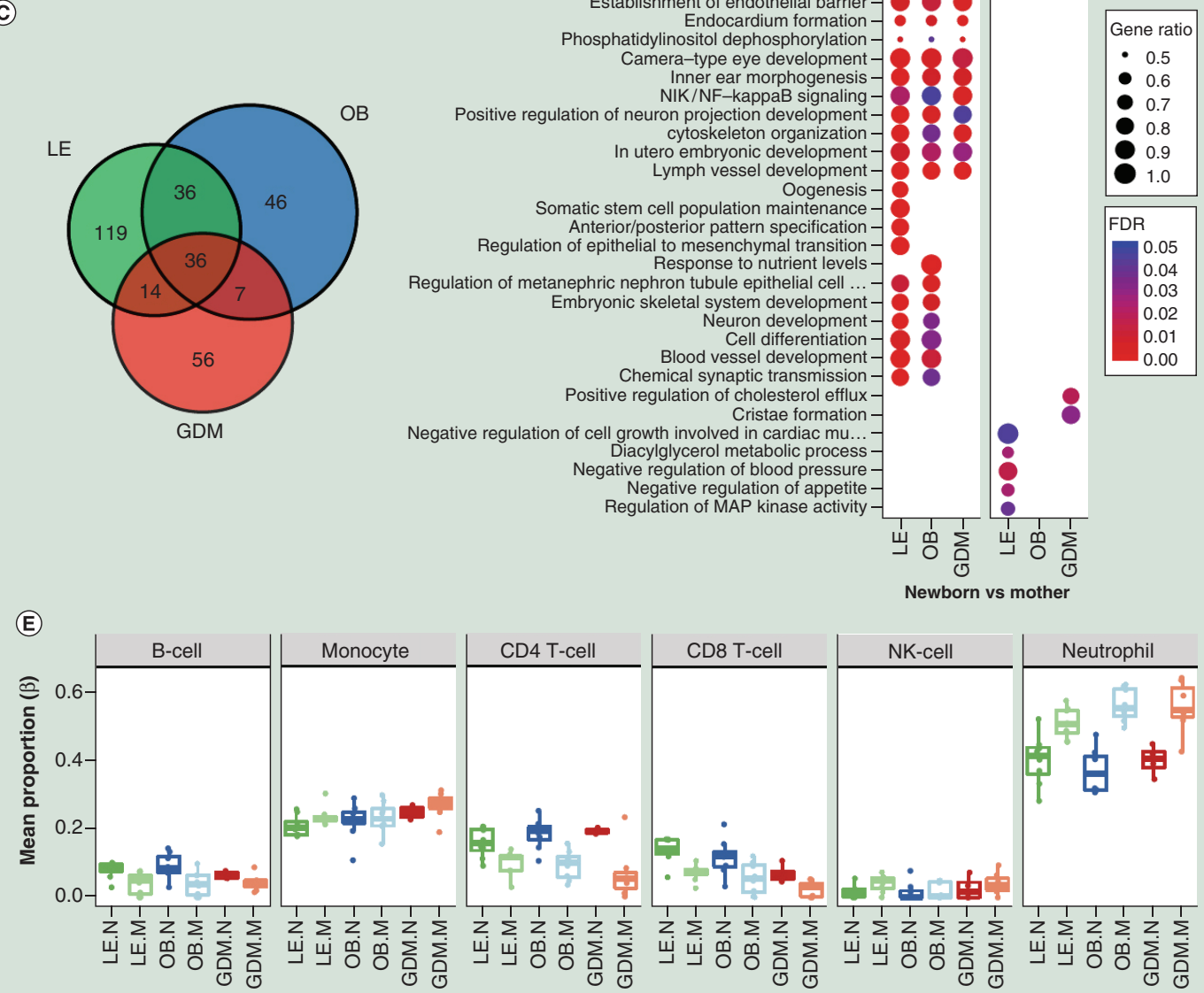

Figure 1. Distinct epigenetic signatures in each mother-newborn pair. (A) MDS plot of leading logFC dimension 1 and 2 of DNA methylation data from whole blood samples removing data from sex chromosomes. (B) Box and whisper plot showing mean CpG methylation levels in individual replicates from LE, OB and GDM across CpG probes. Pairwise Wilcoxon test was used to identify relationships between mother and newborn methylation across groups. The $p$-values were adjusted for multiple testing correction using Benjamini-Hochberg method. (C) Overlap of differential methylated regions of mother-newborn pairs of LE (green), OB (blue) and GDM (red). (D) FRY pathway enrichment for GO biological processes of hypomethylated and hypermethylated genes associated with DMRs of mother-newborn pairs of lean, obese and GDM. Gene ratio was reported as the size of dot and false discovery rate as color as indicated in the legend. (E) Estimated cell populations proportions in $\beta$-value of individual samples between newborn and mother across groups was calculated by EpiDISH.

DMR: Differentially methylated region; GDM: Gestational diabetes mellitus; GO: Gene ontology; LE: Lean; M: Mother; MDS: Multidimensional scaling; N: Newborn; OB: Obese. 
in Lean compared with GDM, and 149 DMRs (125 hypo- and 24 hyper-methylated) comparing Obese to GDM, but no differences between Lean and Obese (Supplementary Table $2 \&$ Figure 2A). Of these DMRs, 73 regions were common (63 hyper- and 10 hypo-methylated) between the comparisons GDM versus Lean and GDM versus Obese (Figure 2B). These data, showing higher association in DNA methylation between mothers compared with newborns, support the idea that a direct transmission of specific DNA methylation signatures from mother to child, without environmental influences, is not the main factor contributing to the distinct DNA methylation signature found in GDM babies.

We sought to investigate if altered epigenetic signatures in GDM newborns were caused by an environmentally driven epigenetic plasticity occurring throughout embryo development and after birth. Recent work suggests that early selection of embryos may influence the nature of the epigenetic signatures of children and, according to such hypothesis, early selection of embryos would lead to a changed variance in DNA methylation in the next generation, whereas epigenetic changes occurring during development and throughout life would drive specific gene expression reprogramming without altering variance [28]. When investigating variance in DMRs in our cohort, we detected higher variance in newborn GDM compared with Lean and Obese newborns, but not in the mothers of either group (Figure 2C). Collectively, our results suggest that environmental factors influence the altered DNA methylation signatures of GDM newborns, and that such signatures may be selected in the early phases of fertilization.

\section{Specific pathways are associated with the altered DNA methylation in newborn with GDM}

Ontology analysis of the genes nearest to the DMRs across newborns, returned enrichment for pathways mainly common between Lean and Obese such as cardiac muscle thin filament assembly for regions hypomethylated, whereas regions with higher DNA methylation levels were enriched for toll signaling pathway and ATP biosynthetic process (Figure 2D \& Supplementary Table 3).

\section{DNA methylation associations with phenotypical outcomes}

To gain insight into the specific clinical factors in mothers that might affect the epigenome of newborns born from GDM mothers, we investigated associations between differential DNA methylation in newborns and clinical features of the mothers. Interestingly, we observed a correlation of five DMRs in newborns with maternal serum values of CRP, HDL and LDL levels, whereas two regions correlated with infant BMI at birth and fat free mass/fat mass at week 12 (Figure 3). We did not replicate these correlations in the mothers (Supplementary Figure 3). On the contrary, we observed that maternal leptin and the phenotypic outcome of the infants correlated to the DNA methylation in specific regions of the mothers (Supplementary Figure 3). These results indicate that DNA methylation at specific regions may predict fat distribution at week 12 and support a role of circulating factors in differentially setting DNA methylation in mothers and in newborns.

\section{Discussion}

In the current study, we compared DNA methylation signatures in blood from mothers with GDM or obesity during pregnancy to that of their respective newborns. We identified that, compared with lean controls, the mother-newborn pairs exhibiting the most differential methylation were from the GDM group. Comparison of DNA methylation signatures in mothers across groups was strikingly similar, suggesting that the altered DNA methylation signatures that we detected in newborns from mothers with GDM are not set by direct transmission, but instead, they were environmentally influenced. The fact that newborns from GDM exhibited the most variance in DNA methylation supports the idea that DNA methylation profiles results mostly from a selection of embryos having a specific epigenome, rather than driven by dynamic epigenetic changes that occurred during in utero development.

The association between DNA methylation signatures in children and the metabolic phenotype of the mother has been investigated in several studies [12-14,20,21,42]. We compared our DNA methylation results to three studies examining the role of GDM on DNA methylation in newborns (chosen because they had genome-wide data publicly available) and found that some regions were commonly differentially methylated in two out of the three studies $[13,14,20]$. The genomic regions found in common between the studies included: four genes common to the comparisons between both Lean and GDM, and Obese and GDM newborns (ACADM, ABCC4, LENG8 and $M B D 3 L 1), 21$ genes common to only the comparison between Lean and GDM newborns (TMCO3, SMYD3, ATG7, G3BP2, ILF3, UHRF1BP1, FGF2, C19orf60, UBTF, PDXK, CPT1B, ERF, TTC9, ZNF837, DLG4, 

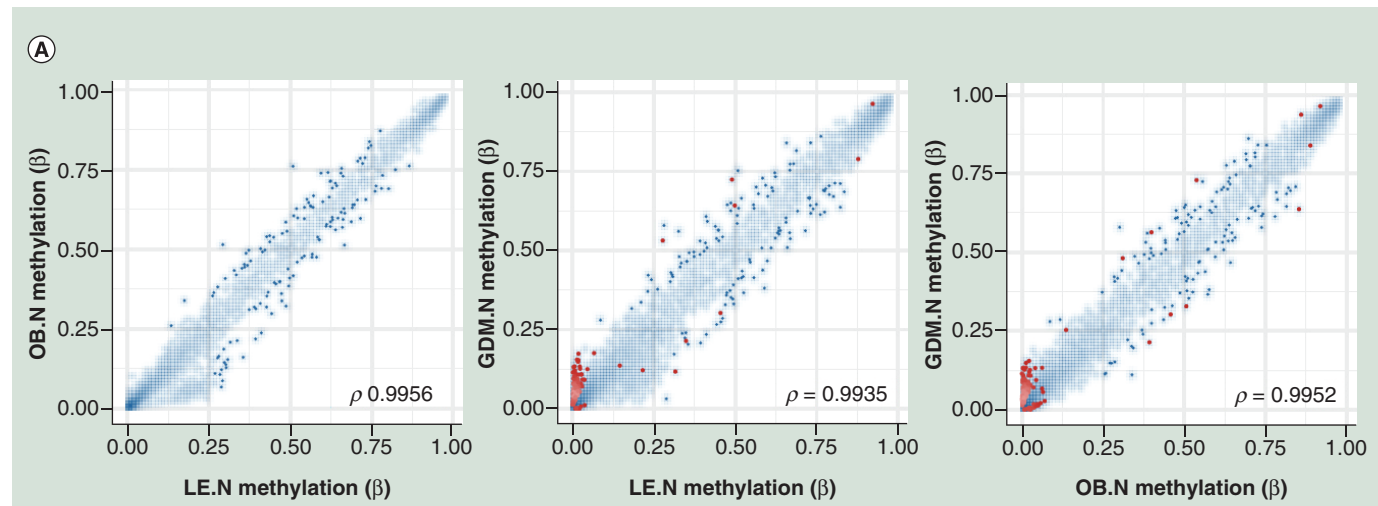

(B)
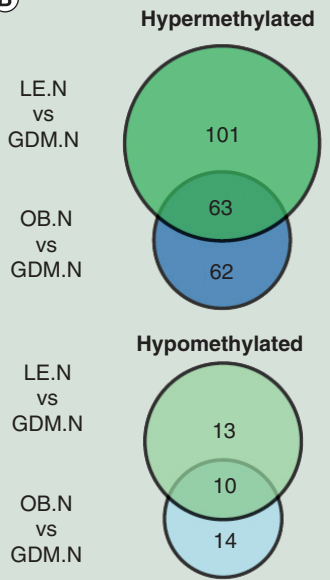

(D)

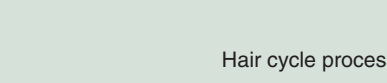

Negative regulation of dephosphorylation Cardiac muscle thin filament assembly Response to angiotensin Positive regulation of apoptotic DNA fragmentationAmyloid-beta clearance by transcytosis

Negative regulation of receptor internalization Cellular response to parathyroid hormone stimulus Establishment of protein localization to membraneMeiotic cell cycleSperm capacitationCristae formation

Regulation of interferon-beta production Response to radiation Toll signaling pathwayNucleosome assembly ATP biosynthetic process Histone H3-K4 trimethylation TRIP-dependent toll-like receptor signaling pathwayNegative regulation of sister chromatid cohesion-

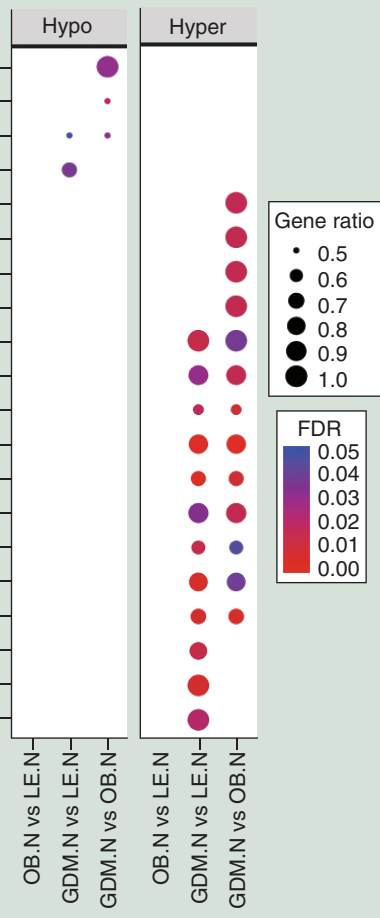

(C)

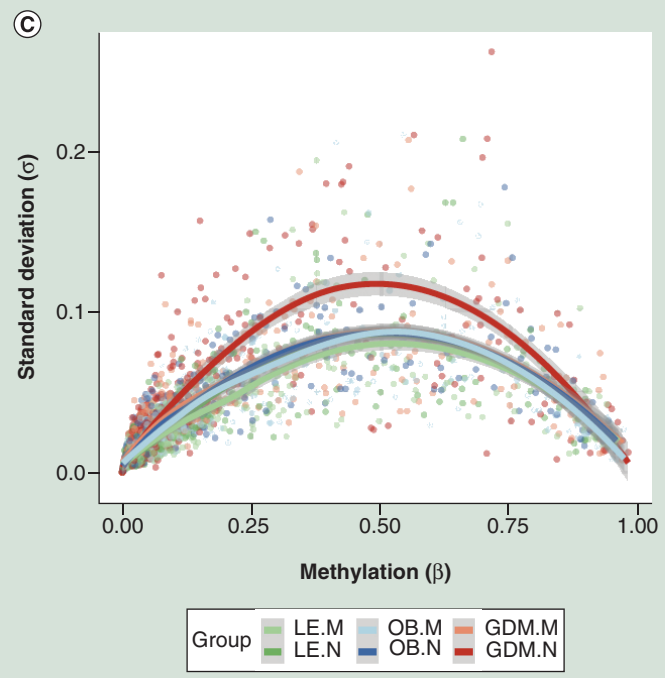

Figure 2. Altered epigenome of gestational diabetes mellitus newborns. (A) Comparison of the $\beta$-methylation of $\mathrm{CpGs}$ in newborns of LE and OB, LE and GDM, and OB and GDM. Red dots represent significant DMRs, faded blue show the smoothed density for CpG probes, while dark blue points show single CpG probes away from dense regions. (B) Overlap of significant DMRs found between newborns of LE versus GDM and OB versus GDM, represented as hypermethylated and hypomethylated DMRs. (C) Relationship between DNA methylation and standard deviation $(\sigma)$ for DMRs found between newborns of lean/obese and GDM. Grey line indicates $95 \%$ Cl. (D) FRY pathway enrichment for GO biological processes of hypomethylated (Hypo) and hypermethylated (Hyper) genes associated with DMRs by comparisons of newborns between LE versus OB, LE versus GDM and OB versus GDM. Gene ratio was reported as the size of dot and false discovery rate as color.

DMR: Differentially methylated region; GDM: Gestational diabetes mellitus; GO: Gene ontology; LE: Lean; N: Newborn; OB: Obese. 

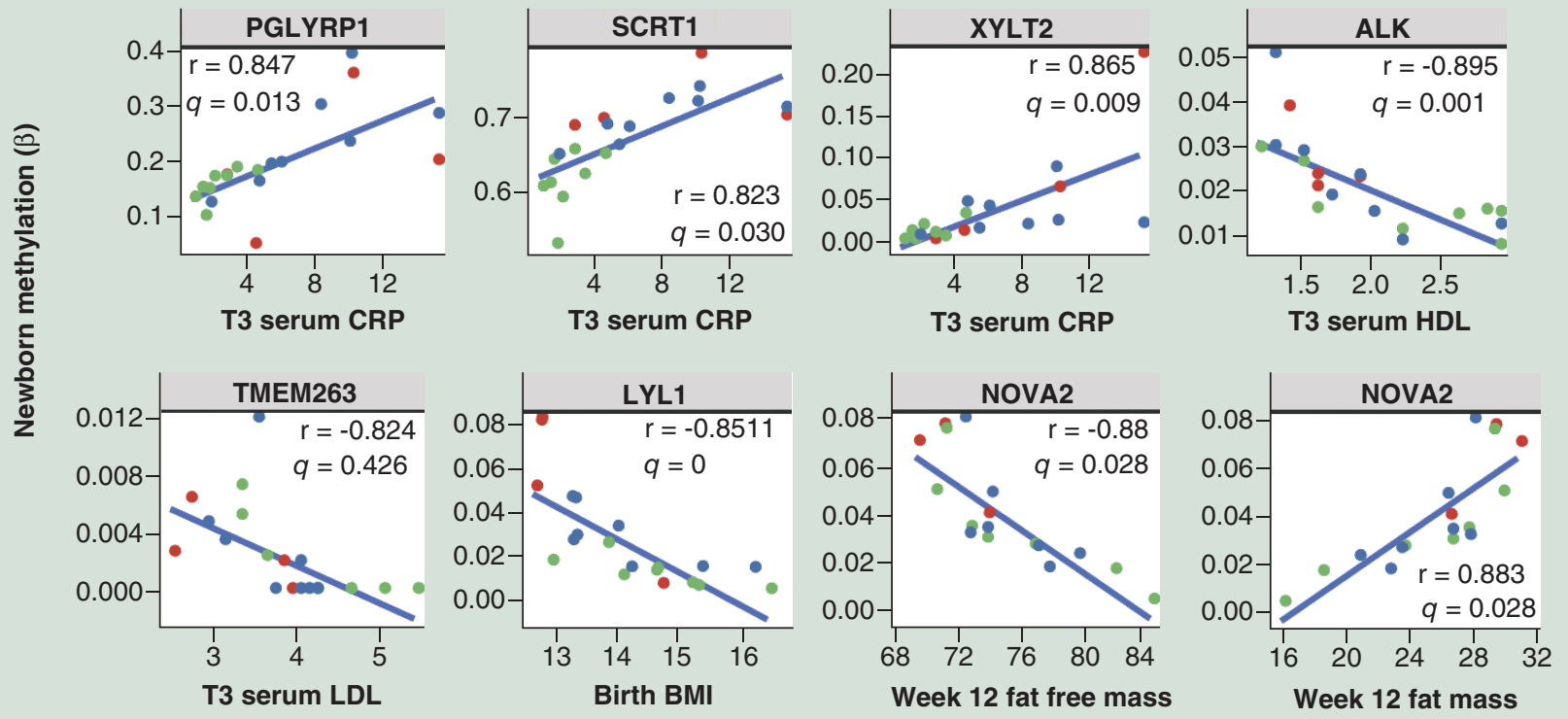

Group $\bullet$ LE $\bullet$ OB $\bullet$ GDM

Figure 3. Correlations between newborn $\beta$-methylation of promoters and clinical parameters. Correlations were performed using Spearman correlation tests. The p-values are adjusted for multiple testing using Benjamini-Hochberg method. CRP: C-reactive protein; GDM: Gestational diabetes mellitus; HDL: High-density lipoprotein; LDL: Low-density lipoprotein; LE: Lean; OB: Obese.

NAP1L4, QTRT1, SLFN11, NPC1, C1orf229 and FOXP1) and five genes common to only the comparison between Obese and GDM newborns (GALK2, XRRA1, TMEM151B, KLF2 and DTNA).

On the contrary, we did not detect $\mathrm{CpG}$ sites in common between our study and the large Pregnancy and Childhood Epigenetics (PACE) consortium study, where 86 sitesassociated with maternal BMI [42]. The number of overlapping genes or DNA methylation regions is quite modest. Such lack of consistency may be caused by the nature of the tissues investigated across studies, for example, either cord blood [13,14,20,21], placenta [12,14,20] or whole blood (current study) [42]. It is also possible that the method used for determining DNA methylation may be introducing some of the inconsistencies across studies. Methodological differences between the Infinium HumanMethylation 450 BeadChips array [12-14,20], pyrosequencing [21] and RRBS (current study) could introduce bias toward distinct genomic regions at various resolution, leading to a discrepancy between studies.

Given that the epigenome is amenable to environmental factors, all the epigenetic marks that we detected in newborns may not be stable throughout life and therefore, may not have any physiological or pathological effect. The notion that most DNA methylation marks are maintained throughout life is suggested by several studies, notably studies investigating DNA methylation signatures in obesity and GDM and which showed that epigenetic signatures detected at birth persist in adolescence $[42,43]$. The PACE consortium identified that the majority $(72$ out of 86) of $\mathrm{CpG}$ sites that were differentially methylated at birth persist in adolescence [42]. Despite the evidence for high maintenance of DNA methylation across life, we did not see any overlap in DNA methylation changes comparing our data from GDM newborns and individuals at 12 years of age from another study [43]. As discussed above, this discrepancy could be caused by cell type specificity or technical bias. Other studies showed that epigenetic marks are under continuous influence of circulating factors throughout life [1-3]. Thus, further studies, using the same technologies for detecting DNA methylation at the whole genome level, should be used at various ages in the same individuals to identify epigenetic marks that are stable throughout life and those under lifestyle influence after birth. Stable epigenetic marks after birth may or may not be stable in utero, and the marks that are under environmental influence in utero but stable after birth represent candidate targets of interest for preventing the effect of adverse developmental programming. 
The major finding in this study was that DNA methylation signatures were similar across mothers from the lean, obese and GDM groups, while DNA methylation signatures were markedly distinct in newborns from GDM mothers. Given our small sample size, our study may not be designed to detect the epigenetic marks that were transmitted through maternal gametic inheritance. However, our results do not support that a direct transmission of epigenetic marks from mother to offspring, through so-called epigenetic inheritance, is the main factor explain the altered epigenetic signatures in GDM children. Instead, our data suggest that environmental factors are the main contributors of altered DNA methylation signatures in GDM newborns. Environmentally influenced epigenetic patterns can be set during embryo development, potentially by a selection of gametes or fertilized embryos harboring specific epigenetic signatures [44]. Such selection may occur based on the gene expression capacity of the developing embryo which, itself, is set by both genetic and epigenetic blueprints. Therefore, to study the effect on the in utero environment versus selection of embryos, it has been postulated that genetic backgrounds should be taken into consideration [6]. Here, the nature of the study design did not allow to correct for genetic influences. Given that GDM has a strong genetic component, we cannot rule out that the epigenetic signature that we detect in offspring from GDM mothers is not caused by selection of embryos of particular genetic makeup which would lead to selection of specific epigenetic profiles. We found that the regions differentially methylated in GDM newborns exhibit higher variance compared with newborns from the lean and the obese groups which, according to several studies, suggests an effect of embryo selection, rather than dynamic epigenetic changes in utero or early after birth $[28,45]$. Similarly, another study showed a tendency toward a higher variance in DNA methylation in GDM, compared with NGT children (mean SD GDM: 0.081; mean SD NGT: 0.075) [20]. If such selection exists in GDM, the clinical parameters specific to GDM which apply selective pressure to embryos remain to be identified. The potential candidates, blood sugar, cholesterol and oxidative stress, which are elevated in gestational diabetes have been implicated in dynamic DNA methylation changes in somatic cells $[22,23,46]$. In the present study, we found that maternal markers at trimester three correlate with DNA methylation at specific regions in the newborns, namely, serum CRP (PGLYRP1, SCRT1 and XYLT2), HDL (ALK) and LDL (TMEM263). These results suggest that a specificity of circulating factors in influencing DNA methylation in utero.

An alternative explanation for not finding DNA methylation signatures that are directly transmitted from mother to children could be the cell type heterogeneity between mother and infant blood samples [41]. Accumulating evidence supports the view that cell type heterogeneity can bias the interpretation of DNA methylation results by misleadingly associating a certain disease or trait to a specific epigenetic mark, while detection of a mark may simply be the consequence of a differential presence of specific cell types each harboring distinct epigenetic signatures [6]. The importance of adjusting for cell heterogeneity was shown previously in the context of GDM, where methylation at only 86 out of $9044 \mathrm{CpG}$ sites originally identified as linked to BMI survived correction for cell-type heterogeneity [42]. In the current study, we did not find differences in cell type composition across mothers or newborns, which does not support the idea that the altered DNA methylation signature in newborns from GDM mothers, compared with obese or lean mothers, is driven by cell type composition. It is possible, however, that slight changes in cell type composition occur in GDM mothers or newborns, thereby masking altered DNA methylation that would be common between mother and newborn and, consequently, affecting our interpretation of the lack of direct transmission from mother to child.

\section{Conclusion}

Our results provide insight into the association between gestational diabetes and the epigenetic signatures of the newborn. Our genome-wide approach identified specific gene pathways that may undergo reprogramming by DNA methylation in newborn of mothers with GDM. If genetic factors are not at play, our computational analyses support the idea that the extracellular milieu during pregnancy is the main factor driving epigenetic changes in the offspring. The pathophysiological consequence of adverse in utero exposure on the epigenome remains to be further investigated.

Supplementary data

To view the supplementary data that accompany this paper please visit the journal website at: www.futuremedicine.com/doi/sup $\mathrm{pl} / 10.2217 /$ epi-2019-0055 


\section{Author contributions}

E Andersen performed experiments, analyzed the data and wrote the manuscript; A Altıntaş performed bioinformatics analysis and generated figures; $U$ Andersson-Hall collected the samples and analyzed the data; A Holmäng designed the study and analyzed the data; R Barrès designed the study, analyzed the data and wrote the manuscript. All authors have approved the final version of the manuscript.

\section{Acknowledgments}

We thank all participating mothers and infants, and past and present members of the PONCH group. We would like to acknowledge The Danish National High-Throughput DNA Sequencing Centre, University of Copenhagen, for sequencing the samples. We would to thank $L$ Small for proof reading the manuscript.

Financial \& competing interests disclosure

The PONCH study is supported by grants from the Swedish Research Council (12206), the Swedish Diabetes Association Research Foundation (2015-08) and the Swedish state under the agreement between the Swedish government and the country councils, the ALF-agreement (720851). The Novo Nordisk Foundation Centre for Basic Metabolic Research is an independent research center at the University of Copenhagen partially funded by an unrestricted donation from the Novo Nordisk Foundation. The authors have no other relevant affiliations or financial involvement with any organization or entity with a financial interest in or a financial conflict with the subject matter or materials discussed in the manuscript apart from those disclosed.

No writing assistance was utilized in the production of this manuscript.

\section{Open access}

This work is licensed under the Attribution-NonCommercial-NoDerivatives 4.0 Unported License. To view a copy of this license, visit http://creativecommons.org/licenses/by-nc-nd/4.0/

\section{Summary points}

- Whole genome DNA methylation profiling of blood samples from lean, obese and gestational diabetes mellitus (GDM) mothers, showed highly similar profiles across groups.

- Analysis of cell type composition between groups showed large differences in neutrophil and T cell composition between mothers and newborns, but only minimal differences across groups.

- Methylation analysis of each mother-newborn pair revealed that epigenetic signatures of newborns from GDM mothers were the most distinct of all the groups.

- Differentially methylated regions between mother-newborn pairs were enriched for genes controlling cholesterol efflux, only in GDM.

- Newborns from GDM mothers have an altered epigenetic profile compared with newborns of both lean and obese mothers.

- Differentially methylated regions in newborns of GDM showed increased variance, suggesting that selection of embryos, rather than environmentally induced epigenetic changes are at play.

- Maternal serum C-reactive protein, high density lipoprotein and low density lipoprotein levels correlate with DNA methylation levels at specific regions, suggesting a role for environmental factors in setting region specific DNA methylation signatures.

- Low level of similarity between mothers and newborns of GDM suggests that environmental factors, rather than direct transmission from mother to child, are involved in establishing the epigenetic signature associated with maternal GDM.

\section{References}

Papers of special note have been highlighted as: $\bullet$ of interest; $\bullet \bullet$ of considerable interest

1. Grunnet LG, Hansen S, Hjort L et al. Adiposity, dysmetabolic traits, and earlier onset of female puberty in adolescent offspring of women with gestational diabetes mellitus: a clinical study within the Danish national birth cohort. Diabetes Care 40(12), 1746-1755 (2017).

2. Sobngwi E, Boudou P, Mauvais-Jarvis F et al. Effect of a diabetic environment in utero on predisposition to Type 2 diabetes. Lancet 361(9372), 1861-1865 (2003).

3. Hughes AE, Nodzenski M, Beaumont RN et al. Fetal genotype and maternal glucose have independent and additive effects on birth weight. Diabetes 67, 1024-1029 (2018).

4. Heijmans BT, Tobi EW, Lumey LH, Slagboom PE. The epigenome: archive of the prenatal environment. Epigenetics 4(8), 526-531 (2009). 
5. Andersson-Hall UK, Järvinen EAJ, Bosaeus MH et al. Maternal obesity and gestational diabetes mellitus affect body composition through infancy: the PONCH study. Pediatr. Res. 85(3), 369-377 (2018).

- Maternal obesity and gestational diabetes increase body fat $\%$ and mass in especially girls of gestational diabetes mellitus mothers.

6. Hannon E, Knox O, Sugden $\mathrm{K}$ et al. Characterizing genetic and environmental influences on variable DNA methylation using monozygotic and dizygotic twins. PLoS Genet. 14(8), 1-27 (2018).

7. Kong A, Thorleifsson G, Frigge ML et al. The nature of nurture: effects of parental genotypes. Science 359(6374), 424-428 (2018).

8. Maurano MT, Wang H, John S et al. Role of DNA methylation in modulating transcription factor occupancy. Cell Rep. 12(7), 1184-1195 (2015).

9. Harris CJ, Scheibe M, Wongpalee SP et al. A DNA methylation reader complex that enhances gene transcription. Science 362(6419), 1182-1186 (2018).

10. Ng RK, Dean W, Dawson C et al. Epigenetic restriction of embryonic cell lineage fate by methylation of Elf5. Nat. Cell Biol. 10(11), 1280-1290 (2008).

11. Sharp AJ, Stathaki E, Migliavacca E et al. DNA methylation profiles of human active and inactive X chromosomes. Genome Res. 21, 1592-1600 (2011).

12. Binder AM, LaRocca J, Lesseur C, Marsit CJ, Michels KB. Epigenome-wide and transcriptome-wide analyses reveal gestational diabetes is associated with alterations in the human leukocyte antigen complex. Clin. Epigenetics 7(1), 1-12 (2015).

13. Weng X, Liu F, Zhang H et al. Genome-wide DNA methylation profiling in infants born to gestational diabetes mellitus. Diabetes Res. Clin. Pract. 142, 10-18 (2018).

14. Finer $\mathrm{S}$, Mathews $\mathrm{C}$, Lowe $\mathrm{R}$ et al. Maternal gestational diabetes is associated with genome-wide DNA methylation variation in placenta and cord blood of exposed offspring. Hum. Mol. Genet. 24(11), 3021-3029 (2014).

15. Yang IV, Zhang W, Davidson EJ, Fingerlin TE, Kechris K, Dabelea D. Epigenetic marks of in utero exposure to gestational diabetes and childhood adiposity outcomes: the EPOCH study. Diabet. Med. 35(5), 612-620 (2018).

16. Kim E, Kwak SH, Chung HR et al. DNA methylation profiles in sibling pairs discordant for intrauterine exposure to maternal gestational diabetes. Epigenetics 12(10), 825-832 (2017).

17. Díaz M, García C, Sebastiani G, de Zegher F, López-Bermejo A, Ibáñez L. Placental and cord blood methylation of genes involved in energy homeostasis: association with fetal growth and neonatal body composition. Diabetes 66(3), 779-784 (2017).

18. Kang J, Lee C-N, Li H-Y, Hsu K-H, Lin S-Y. Genome-wide DNA methylation variation in maternal and cord blood of gestational diabetes population. Diabetes Res. Clin. Pract. 132, 127-136 (2017). www.sciencedirect.com/science/article/pii/S0168822717304795

19. Cardenas A, Gagné-ouellet V, Allard C et al. Placental DNA methylation adaptation to maternal glycemic response in pregnancy. Diabetes 67(August), 1673-1683 (2018).

20. Ruchat SM, Houde AA, Voisin G et al. Gestational diabetes mellitus epigenetically affects genes predominantly involved in metabolic diseases. Epigenetics 8(9), 935-943 (2013).

21. El Hajj N, Pliushch G, Schneider E et al. Metabolic programming of MEST DNA methylation by intrauterine exposure to gestational diabetes mellitus. Diabetes 62(4), 1320-1328 (2013).

22. Barrès R, Osler ME, Yan $\mathrm{J}$ et al. Non-CpG methylation of the PGC-1alpha promoter through DNMT3B controls mitochondrial density. Cell Metab. 10, 189-198 (2009).

23. Andersen E, Ingerslev LR, Fabre $\mathrm{O}$ et al. Preadipocytes from obese humans with Type 2 diabetes are epigenetically reprogrammed at genes controlling adipose tissue function. Int. J. Obes. 43, 306-318 (2019).

24. Fabre O, Ingerslev LR, Garde C, Donkin I, Simar D, Barrès R. Exercise training alters the genomic response to acute exercise in human adipose tissue. Epigenomics 10(8), 1033-1050 (2018).

25. Barrès $\mathrm{R}$, Kirchner $\mathrm{H}$, Rasmussen $\mathrm{M}$ et al. Weight loss after gastric bypass surgery in human obesity remodels promoter methylation. Cell Rep. 3, 1020-1027 (2013).

26. Barrès R, Yan J, Egan B et al. Acute exercise remodels promoter methylation in human skeletal muscle. Cell Metab. 15(3), 405-411 (2012).

27. Jacobsen SC, Brøns C, Bork-Jensen J et al. Effects of short-term high-fat overfeeding on genome-wide DNA methylation in the skeletal muscle of healthy young men. Diabetologia 55(12), 3341-3349 (2012).

28. Tobi EW, van den Heuvel J, Zwaan BJ, Lumey LH, Heijmans BT, Uller T. Selective survival of embryos can explain DNA methylation signatures of adverse prenatal environments. Cell Rep. 25(10), 2660-2667.e4 (2018).

-• Mathematically suggests that adverse prenatal environments may drive changes in DNA methylation signatures by altered selection of embryos.

29. Lind T, Phillips PR. Influence of pregnancy on the 75-g OGTT: a prospective multicenter study. Diabetes 40(2), 8-13 (1991).

30. Krueger F, Andrews SR. Bismark: a flexible aligner and methylation caller for Bisulfite-Seq applications. Bioinformatics 27(11), 1571-1572 (2011). 
31. Hastie AT, Tibshirani R, Narasimhan B. impute: impute: imputation for microarray data. R package version 1.56.0., Feb 12, 1-5 (2018).

32. Hebestreit K, Dugas M, Klein HU. Detection of significantly differentially methylated regions in targeted bisulfite sequencing data. Bioinformatics 29(13), 1647-1653 (2013).

33. Chen Y, Pal B, Visvader JE, Smyth GK. Differential methylation analysis of reduced representation bisulfite sequencing experiments using edgeR [version 1; referees: 2 approved, 1 approved with reservations]. F1000Res 6(2055), 1-40 (2017).

34. Chen Y, Lun ATL, Smyth GK. From reads to genes to pathways: differential expression analysis of RNA-Seq experiments using Rsubread and the edgeR quasi-likelihood pipeline. F1000Res 5, 1438 (2016).

35. Benjamini Y, Hochberg Y. Controlling the false discovery rate: a practical and powerful approach to multiple testing. J. R. Stat. Soc. B 57(1), 289-300 (1995).

36. Martens JHA, Stunnenberg HG. BLUEPRINT: mapping human blood cell epigenomes. Haematologica 98(10), 1487-1489 (2013).

37. Albrecht F, List M, Bock C, Lengauer T. DeepBlueR: large-scale epigenomic analysis in R. Bioinformatics 33(13), 2063-2064 (2017).

38. Zou H, Trevor H, Tibshirani R. Sparse principal component analysis. J. Comput. Graph. Stat. 15(2), $265-286$ (2006).

39. Houseman EA, Accomando WP, Koestler DC et al. DNA methylation arrays as surrogate measures of cell mixture distribution. $B M C$ Bioinformatics 13(1), 86 (2012).

40. Teschendorff AE, Breeze CE, Zheng SC, Beck S. A comparison of reference-based algorithms for correcting cell-type heterogeneity in epigenome-wide association studies. BMC Bioinformatics 18(105), 1-14 (2017).

41. Valiathan R, Ashman M, Asthana D. Effects of ageing on the immune system: infants to elderly. Scand. J. Immunol. 83(4), 255-266 (2016).

42. Sharp GC, Salas LA, Monnereau C et al. Maternal BMI at the start of pregnancy and offspring epigenome-wide DNA methylation: findings from the pregnancy and childhood epigenetics (PACE) consortium. Hum. Mol. Genet. 26(20), 4067-4085 (2017).

-. Demonstrates that maternal BMI affect DNA methylation in a large multicenter study. BMI of the mother is associated with altered DNA methylation at 86 sites in newborns.

43. Hjort L, Martino D, Grunnet LG et al. Gestational diabetes and maternal obesity are associated with epigenome-wide methylation changes in children. JCI Insight 3(17), 1-14 (2018).

- Gestational diabetes and maternal obesity are associated with changes in the DNA methylation of 9-16-year old children.

44. Donkin I, Barrès R. Sperm epigenetics and influence of environmental factors. Mol. Metab. 14, 1-11 (2018).

-. Reviews the effect of environmental factors on epigenetic inheritance.

45. Tobi EW, Slieker RC, Stein AD et al. Early gestation as the critical time-window for changes in the prenatal environment to affect the adult human blood methylome. Int. J. Epidemiol. 44(4), 1211-1223 (2015).

46. Zhang YW, Wang Z, Xie W et al. Acetylation enhances TET2 function in protecting against abnormal DNA methylation during oxidative stress. Mol. Cell 65(2), 323-335 (2017). 
